\title{
MS40-04 I In Situ X-RAy SCATtering Study of Hydrothermal Synthesis of Anatase TiO2 Nanoparticles from Commercial Precursor TiOSO 4
}

Søndergaard-Pedersen, Frederik (Aarhus University, Aarhus C, DNK); Broge, Nils Lau Nyborg (Aarhus University, Aarhus C, DNK); Yu, Jinlong (Aarhus University, Aarhus C, DNK); Mamakhel, Aref (Aarhus University, Aarhus C, DNK); Beyer, Jonas (Aarhus University, Aarhus C, DNK); Brummerstedt Iversen, Bo (Aarhus University, Aarhus, DNK)

$\mathrm{TiO}_{2}$ is one of the most important metal oxides in modern materials science. Due to its low cost and non-toxicity it is very attractive in applications such as water splitting, photocatalysis and as a support material for catalytically active species e.g. in selective catalytic reduction of NOx gases.

Hydrothermal synthesis is an environmentally benign method for obtaining nanoparticles of TiO2, which allows easy upscaling and tailoring of the products. Rutile $\left(\mathrm{P}_{2} / \mathrm{mnm}\right)$ is the thermodynamically stable bulk $\mathrm{TiO}_{2} \mathrm{phase}$ but anatase $\left(14_{1} / \mathrm{amd}\right)$ is usually observed as the initial phase in hydrothermal synthesis of $\mathrm{TiO}_{2}$, which has been explained in terms of anatase having a lower surface energy than rutile. This causes anatase to have a lower overall free energy at small particle sizes until a certain critical size is reached at which rutile becomes the most stable phase.

Most academic $\mathrm{TiO}_{2}$ studies start from precursors that are very expensive and/or highly reactive (e.g. titanium alkoxides, $\mathrm{TiCl}_{4}$ and titanium metal). This makes the procedures unsuitable for upscaling to industrial production thus preventing their use in real world applications. $\mathrm{TiOSO}_{4}$ is an alternative precursor, which is cheap, easy to handle and relatively safe. Hydrothermal treatment of $\mathrm{TiOSO}_{4}$ is observed to yield anatase nanoparticles that are larger than the critical size. To understand why, in situ observations of the nucleation and growth processes are necessary. Findings using a capillary setup which allows measurement of X-ray powder diffraction and total scattering during hydrothermal synthesis will be presented. 